\title{
Immunoprivileged status of the liver is controlled by Toll-like receptor 3 signaling
}

Karl S. Lang, ${ }^{1}$ Panco Georgiev, ${ }^{2}$ Mike Recher, ${ }^{1}$ Alexander A. Navarini, ${ }^{1}$ Andreas Bergthaler, ${ }^{1}$ Mathias Heikenwalder, ${ }^{3}$ Nicola L. Harris, ${ }^{1}$ Tobias Junt, ${ }^{1}$ Bernhard Odermatt, ${ }^{1}$ Pierre-Alain Clavien, ${ }^{2}$ Hanspeter Pircher, ${ }^{4}$ Shizuo Akira, ${ }^{5}$ Hans Hengartner, ${ }^{1}$ and Rolf $M$. Zinkernagel ${ }^{1}$

${ }^{1}$ Institute of Experimental Immunology, ${ }^{2}$ Department of Visceral and Transplantation Surgery, and ${ }^{3}$ Department of Neuropathology, University Hospital of Zurich, Zurich, Switzerland. ${ }^{4}$ Department of Immunology, University Hospital Freiburg, Freiburg, Germany. 5Department of Host Defense, Osaka University, Suita, Japan.

\begin{abstract}
The liver is known to be a classical immunoprivileged site with a relatively high resistance against immune responses. Here we demonstrate that highly activated liver-specific effector $\mathrm{CD}^{+} \mathrm{T}$ cells alone were not sufficient to trigger immune destruction of the liver in mice. Only additional innate immune signals orchestrated by TLR3 provoked liver damage. While TLR3 activation did not directly alter liver-specific $\mathrm{CD8}^{+} \mathrm{T}$ cell function, it induced IFN- $\alpha$ and TNF- $\alpha$ release. These cytokines generated expression of the chemokine CXCL9 in the liver, thereby enhancing $\mathrm{CD}^{+} \mathrm{T}$ cell infiltration and liver disease in mice. Thus, nonspecific activation of innate immunity can drastically enhance susceptibility to immune destruction of a solid organ.
\end{abstract}

\section{Introduction}

The liver represents a so-called immunoprivileged site according to its relative resistance against organ rejection after transplantation (1). This is in contrast to the rapid rejection of purified transplanted allogenic hepatocytes in vivo (2). Also, autoimmune hepatitis due to attack by $B$ and $T$ cells is a relatively rare manifestation of autoimmune disease $(3,4)$. Interestingly, diagnostic markers of autoimmune hepatitis such as antimitochondrial antibodies are also found in healthy people (5). Together, these findings suggest that there exist mechanisms protecting immune attack against this solid organ. Some studies of immune reactivity against components of solid peripheral organs (such as pancreatic islet cells or salivary gland or thyroid antigens) indicate that self-reactive effector $\mathrm{T}$ or B cells alone may not be sufficient for disease induction without additional "inflammatory signals" being required for efficient induction of disease (6). Consistent with clinical observations, results of studies in animal models suggest that naive liverreactive $\mathrm{T}$ cells ignore the liver antigen (7) or become tolerized within the liver $(8,9)$. Inflammation (e.g., infection with systemic bacteria) may upregulate costimulatory molecules in the liver and can break this tolerance $(10,11)$.

Besides priming of an adaptive immune response, viruses can promote inflammatory signals through their ability to activate the innate immune system via TLRs (12). Recently it was shown that activation of TLR3 or TLR7 (which recognize double-stranded and single-stranded RNA [dsRNA and ssRNA], respectively) promotes autoimmunity in mice exhibiting high frequencies of functional autoreactive $\mathrm{CD}^{+} \mathrm{T}$ cells. Disease onset/progression closely correlated with IFN- $\alpha$ production $(13,14)$, suggesting that TLR-induced production of proinflammatory cytokines such as IFN- $\alpha$ and TNF- $\alpha$ may influence the development of autoimmunity.

Nonstandard abbreviations used: ALT, alanine amino-transferase; CXCL9, CXC chemokine ligand 9; IL-7R $\alpha$, IL-7 receptor $\alpha$; LCMV, lymphocytic choriomeningitis virus; poly(I:C), polyinosinic-polycytidylic acid; PTX, pertussis toxin.

Conflict of interest: The authors have declared that no conflict of interest exists. Citation for this article: J. Clin. Invest. 116:2456-2463 (2006). doi:10.1172/JCI28349.
Here we analyzed the requirements of autoimmune liver destruction in a mouse model where the lymphocytic choriomeningitis virus-glycoprotein ${ }_{1-60}$ (LCMV-glycoprotein ${ }_{1-60}$ ) is expressed in the liver as a transgene under the control of the mouse albumin promoter (Alb-1 mice) (7).

\section{Results}

Requirement of TLR3 signaling for destructive autoimmunity. We injected splenocytes from TCR-Tg 318 mice in which $50 \%$ of the CD8 ${ }^{+}$ $\mathrm{T}$ cells express a TCR recognizing the LCMV-glycoprotein ${ }_{33-41}$ epitope presented on $\mathrm{H}-2 \mathrm{D}^{\mathrm{b}} \mathrm{MHC}$ class I molecules into Alb-1 mice or control C57BL/ 6 mice. Without further treatment, those $\mathrm{T}$ cells virtually ignored the antigen in the liver and did not further expand, with no signs of liver damage (Figure 1A and Supplemental Figure 1; supplemental material available online with this article; doi:10.1172/JCI28349DS1) (7). Infection of such recipient mice with 200 PFU of the LCMV strain WE (LCMV-WE) caused massive expansion of gp33-specific $\mathrm{CD}^{+} \mathrm{T}$ cells, as detected by gp33 tetramer binding (7) (Figure 1B), and resulted in liver injury as determined by increased serum activity of alanine amino-transferase (ALT) and high bilirubin serum concentration (7) (Figure 1B). In this experimental model, LCMV induces both a strong adaptive $T$ cell response against liver antigens and a strong innate immune response. To separately analyze the influence of this innate activation, we immunized mice with gp33 peptide together with the TLR9 ligand CPG (ODN-1826). This treatment resulted in a similar expansion of tetramer-positive $T$ cells but only a small increase in ALT and undetectable bilirubin in the serum (Figure 1C). CPG (ODN-1826) is a ligand for TLR9, and its binding to TLR9 results in DC activation and upregulation of costimulatory molecules (15) but does not induce massive IFN- $\alpha$ production. In contrast, LCMV, CpG (ODN-2216), and the RNA repeat polyinosinic-polycytidylic acid [poly(I:C)] activate innate immunity and result in production of IFN- $\alpha(16,17)$. We therefore tested whether poly(I:C) could mimic the ability of replicating LCMV in promoting liver disease. Mice adoptively transferred with autoreactive TCR-Tg 318 CD8 $^{+} \mathrm{T}$ cells were immunized on days 0 and 4 with gp33 plus CpG (ODN-1826). Peptide priming without 

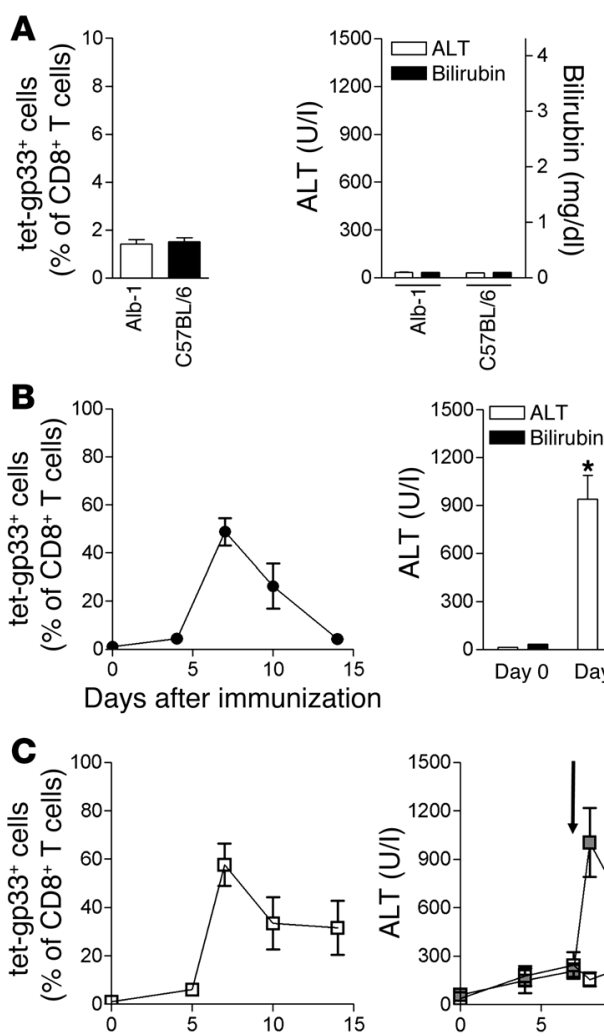

Days after immunization
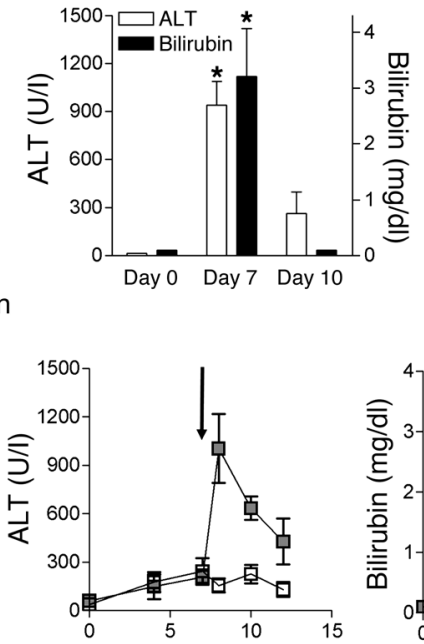

Days after immunization

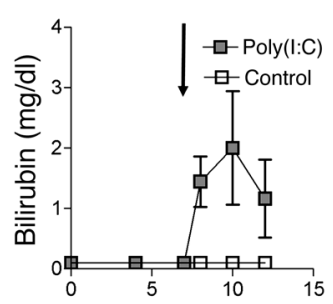

Days after immunization

\section{Figure 1}

TLR3 ligation converts autoreactivity into autoimmune disease. $10^{7}$ splenocytes from LCMV-gp33/ $\mathrm{H}-2 \mathrm{D}^{\mathrm{b}}$-specific TCR-Tg 318 mice were injected i.v. into naive Alb-1 mice or control C57BL/6 mice on day -1. (A) Seven days after transfer, mice were analyzed for gp33-specific T cells and for liver enzymes. Proliferation of transferred cells was analyzed with CFSE (Supplemental Figure 1). (B) After transfer of 318 splenocytes, Alb-1 mice were infected with 200 PFU LCMV-WE on day 0 (filled circles), and numbers of tet-gp33+ CD8 $+\mathrm{T}$ cells determined in peripheral blood over time. Serum activities of ALT and bilirubin serum concentrations were also determined $(n=5)$. ${ }^{*} P>0.05$. (C) Mice were immunized with gp33 (1 mg in PBS) and $\mathrm{CpG}$ on days 0 and 4 (open squares). A separate group of mice was additionally treated with poly $(\mathrm{I}: \mathrm{C})$ on day 7 (arrows) (shaded squares). Numbers of blood tet-gp33 ${ }^{+} \mathrm{CD} 8{ }^{+} \mathrm{T}$ cells, serum ALT activities, and bilirubin serum concentrations were determined ( $n=5-7$ per group).
CpG (ODN-1826) also expanded TCR-Tg CD8 ${ }^{+} \mathrm{T}$ cells (data not shown); however, gp33 plus CPG (ODN-1826) yielded maximal $\mathrm{T}$ cell responses. After priming of TCR-Tg CD8 ${ }^{+} \mathrm{T}$ cells, 1 group of mice was additionally treated with poly(I:C) on day 7 . Administration of poly(I:C) resulted in a rapid elevation of ALT and bilirubin in the serum, indicating hepatocyte destruction (Figure 1C). In separate experiments, peptide-treated mice were further treated with the IFN- $\alpha$-inducing CpG (ODN-2216) on day 7. This activation of TLR9 resulted in a mild but significant increase in ALT, suggesting that other TLRs may also be able to trigger hepatitis (Supplemental Figure 2).

Induction of type I interferons can result in upregulation of MHC class I molecules on pancreatic islet cells, which correlates with onset of autoimmune diabetes $(14,18)$. In contrast, hepatocytes have been described as constitutively expressing abundant surface MHC class I (19), which was not further affected by interferon (Supplemental Figure 3). Thus, antigen presentation on MHC class I molecules did not explain the observed differences in immunologically induced liver destruction.

TLR3-independent $C D 8^{+} T$ cell effector function. Our findings might be explained by enhanced $\mathrm{CD}^{+} \mathrm{T}$ cell function as a consequence of TLR3 engagement. To investigate this possibility, we transfused naive C57BL/6 mice with $10^{7}$ gp33-specific TCR-Tg CD8 ${ }^{+} \mathrm{T}$ cells and immunized recipient mice with gp33 plus $\mathrm{CpG}(\mathrm{ODN}-1826)$ on days 0 and 4, with or without additional treatment with poly(I:C) on day 7. Thereafter, autoreactive $\mathrm{CD}^{+} \mathrm{T}$ cells were identified by tetramer binding and their expression of various activation markers analyzed by FACS analysis. First we analyzed the early activation markers CD25 and CD69. There was a weak expression of CD25 in both groups (Figure 2A). During treatment with poly(I:C), a subset of gp33-specific CD8 T cells upregulated CD69, known to be a target molecule of type I interferon (20). $\mathrm{CD}^{+} \mathrm{T}$ cells from either control or poly(I:C)-treated mice showed no difference in other activation markers (Figure 2B), nor were any differences observed in the ability of these cells to produce IFN- $\gamma$ or to express granzyme B following in vitro restimulation with gp33 peptide (Figure $2 \mathrm{C})$. To examine whether poly(I:C) affected effector function of $\mathrm{CD}^{+} \mathrm{T}$ cells, we performed in vitro and in vivo killing assays. Both mice primed with peptide and CpG (ODN-1826) alone and mice additionally treated with poly(I:C) generated effector $\mathrm{CD}^{+}$ $\mathrm{T}$ cells capable of lysing gp33-labeled target cells in vivo and in vitro (Figure 2, D and E). Staining of the effector protein granzyme B directly ex vivo revealed no significant difference between tet-gp $33^{+} \mathrm{T}$ cells treated with and without poly(I:C) (Figure 2F). Recently it has been shown that $\mathrm{CD}^{+} \mathrm{T}$ cells can express FoxP3 and have regulatory function (21). Peptide priming might also induce a regulatory status in gp33-specific CD8 T cells. However, we did not observe expression of FoxP3 in gp33-specific cells (Supplemental Figure 4). We found that in both C57BL/6 and Alb-1 mice, $\mathrm{CD} 4{ }^{+} \mathrm{CD} 25^{+} \mathrm{T}$ cells were present in the liver after LCMV infection (Supplemental Figure 4). Therefore, we examined whether peptide treatment followed by poly(I:C) treatment had an effect on frequencies of Tregs. While peptide treatment did not result in any significant change in $\mathrm{CD} 4^{+}$Tregs, we have monitored a slight increase in $\mathrm{CD}_{25} 5^{+} \mathrm{FoxP} 3{ }^{+} \mathrm{CD} 4^{+} \mathrm{T}$ cells after poly(I:C) treatment in the liver. We therefore concluded that TLR3 signals did not induce hepatitis by directly altering liver-specific $\mathrm{CD}^{+} \mathrm{T}$ cell function or by downregulation of Treg subsets.

Effects of TLR 3 ligation on liver gene expression. To determine factors responsible for poly(I:C)-induced autoimmunity, we performed 

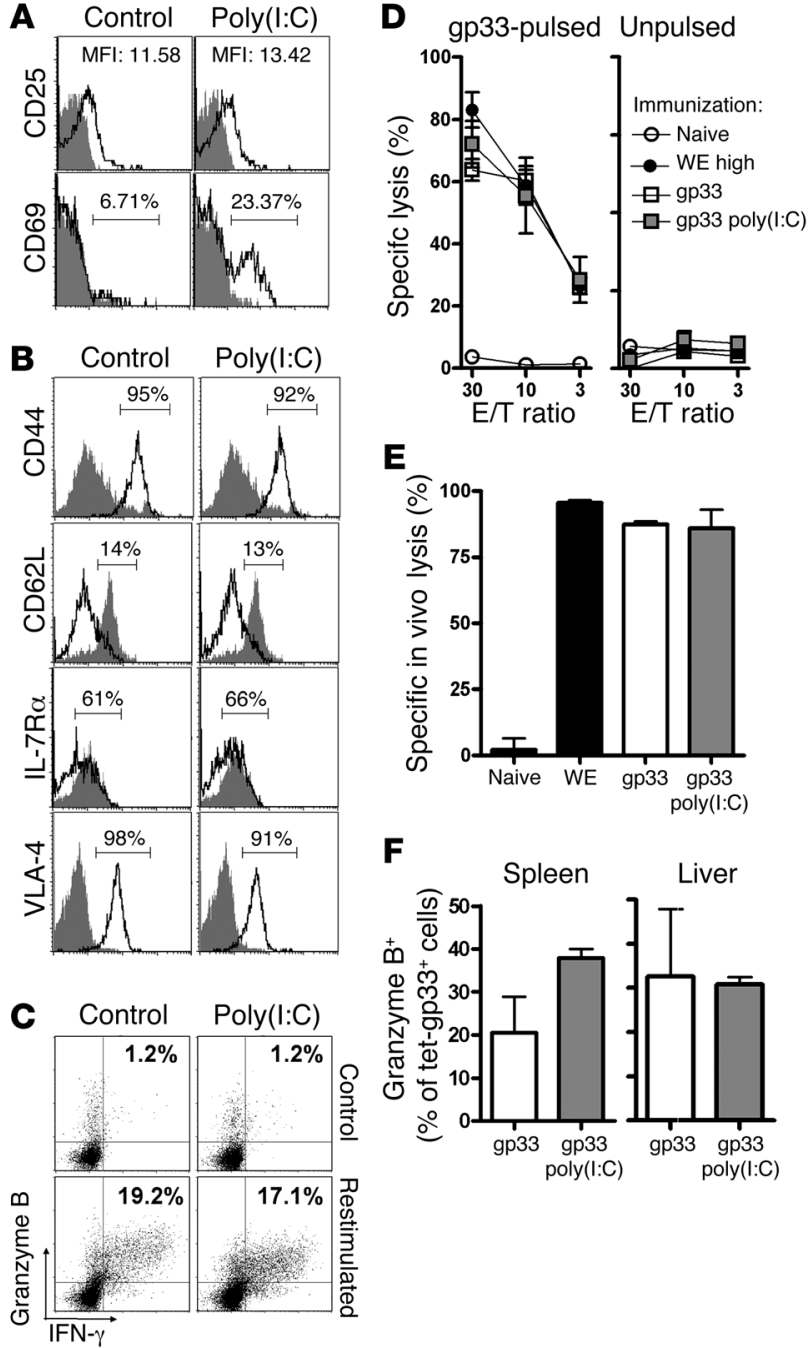

E

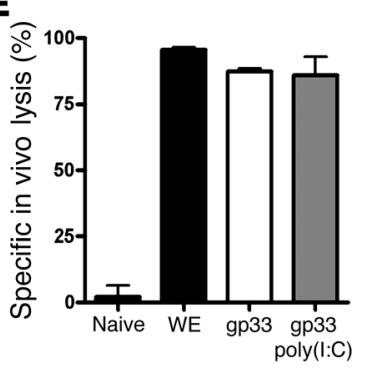

$\mathbf{F}$

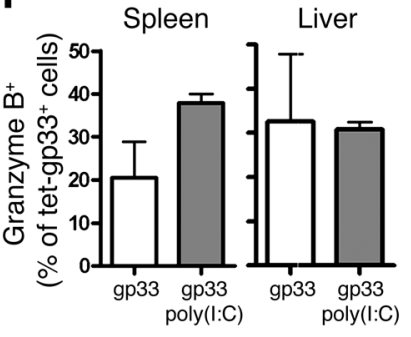

\section{Figure 2}

No influence of TLR3 ligation on CD8 ${ }^{+} \mathrm{T}$ cell effector function. $10^{7}$ splenocytes from LCMV-gp33/H-2D ${ }^{\text {b}}$-specific TCR-Tg 318 mice were injected i.v. into naive C57BL/6 mice. Mice were immunized with gp33 ( $1 \mathrm{mg}$ in PBS) and $\mathrm{CpG}$ on days 0 and 4 . A separate group of mice was additionally treated with poly $(\mathrm{I}: \mathrm{C})$ on day 7 . The phenotype and function of $\mathrm{CD}^{+} \mathrm{T}$ cells were analyzed 24 hours (A-E; $n=3$ per group) or 12 hours (F; $n=2-3$ per group) later. (A) Splenocytes were analyzed by FACS for early activation marker CD25 and CD69. Histogram plots show cells gated on CD8 and Thy 1.1 (marker for $318 \mathrm{~T}$ cells). Gray shading indicates staining with isotype control antibody. Values for FACS analysis give mean fluorecence intensity (MFI). For other plots in A, B, and C, the number of positive expressing cells (marked by bar or quadrant) are given. (B) Splenocytes were further analyzed for surface expression of CD44, CD62L, IL-7R $\alpha$, and VLA-4 (CD49d). Histogram plots show cells gated for CD8 expression and tet-gp33 expression (indicated by a black line) or tet-gp33-negative cells (control expression, indicated by gray shading). (C) Splenocytes were restimulated in vitro with or without gp33, then analyzed for intracellular expression of granzyme B and IFN- $\gamma$. Dot plots show cells gated for CD8 expression. (D and E) Splenocytes were analyzed for their ability to lyse peptideloaded target cells in vitro (D) or in vivo (E). Naive splenocytes served as negative control; splenocytes from mice infected with $2 \times 10^{6} \mathrm{pfu}$ of LCMV-WE (WE high) served as positive control. (F) Eight hours after treatment with poly $(\mathrm{l}: \mathrm{C})$, splenocytes were analyzed for their expression of granzyme $\mathrm{B}(n=2-3)$.

(data not shown). The ability to upregulate CXCL9 expression was TLR3 dependent. This was confirmed by poly(I:C) treatment of $T l r 3^{-/}$mice, which exhibited reduced upregulation (Figure 3B). This effect was mediated through TLR3 ligation on bone marrowderived cells but not on hepatocytes, as $\mathrm{Tlr}^{-{ }^{--}}$bone marrow transferred into irradiated TLR3-competent mice $\left(T l r 3^{-1-} \rightarrow\right.$ BALB/c) yielded a reduced $\mathrm{Cxcl} 9$ expression similar to that in control $\mathrm{Tlr}^{-1-}$ mice. TLR3-competent bone marrow transferred into irradiated $\mathrm{Tlr}^{-/-}$mice $\left(\mathrm{BALB} / \mathrm{c} \rightarrow \mathrm{Tlr}^{-/-}\right.$) resulted in $\mathrm{Cxcl}$ l9 expression similar to that in wild-type control mice (Figure 3B). Loss of TLR3 did not completely abolish poly(I:C)-induced $\mathrm{Cxcl}$ (9) expression, possibly due to the activation of other molecules, e.g., retinoic acid-inducible gene I (RIG-I), which can recognize dsRNA (22). To analyze the role of TLR3-induced cytokines such as interferons and TNF- $\alpha$ in upregulation of Cxcl9 and Vcam-1, mice deficient in receptors for type I or type II interferons or TNF- $\alpha$ were employed. Both IFN- $\alpha$ and TNF- $\alpha$ were produced following poly(I:C) administration (Table 1 and Supplemental Figure 5), and the absence of receptors for either factor abolished the ability of poly(I:C) to induce $\mathrm{Cxcl}$ l9 upregulation (Figure 3C). No role for type II interferons (IFN- $\gamma$ ) was found, since mice deficient in interferon type II receptors (Ifngr ${ }^{--}$) revealed responses comparable to those in wild-type mice following poly(I:C) treatment (Figure 3C). Vcam-1 expression appeared to be regulated solely by TNF- $\alpha$, with no obvious role observed for either type I or type II interferons (Figure $3 \mathrm{D})$. Taken together, the results suggest that TLR3 engagement on bone marrow-derived cells changed liver expression of $\mathrm{T}$ cell-attracting molecules.

Enhanced $C D 8^{+} T$ cell recruitment into the liver by TLR 3 ligation. The upregulation of genes involved in $\mathrm{T}$ cell homing prompted us to investigate whether poly(I:C) treatment resulted in increased $\mathrm{T}$ cell migration into the liver in vivo. It was likely that CXCL9 could attract memory $\mathrm{T}$ cells, as most of the $\mathrm{CD} 44^{+} \mathrm{CD} 8^{+} \mathrm{T}$ cells in C57BL/ 6 mice expressed its receptor CXCR3 (Figure 3E). Treatment with poly (I:C) diminished staining of CXCR3 on memory 
Table 1

mRNA profile of livers 24 hours after treatment with poly(l:C)

\begin{tabular}{|c|c|c|c|c|c|c|c|}
\hline $\begin{array}{l}\text { Fold } \\
\text { difference }\end{array}$ & $\begin{array}{c}\text { Homing/ } \\
\text { inflammation }\end{array}$ & T cell survival & Apoptotic & Antiapoptotic & Signaling & Antiviral & Others \\
\hline $10-100$ & $\mathrm{Cxc} / 9$ & & & & & & \\
\hline $2-10$ & $\begin{array}{l}\text { Tnf- } \alpha, \text { Vcam-1, } \\
\text { Cxcl10, Icam-1A }\end{array}$ & $11-7$ & Fas-ligand & & Irf-7 & $M x-1$, Usp18 & II-10, Igfbp5 \\
\hline $0.5-2$ & $\|-1 \alpha$ & $\|-15\|-18$, & $\begin{array}{c}\text { Bax, Traf6, } \\
\text { Tnf-receptor I, } \\
\text { caspase 8, Fas, } \\
\text { caspase } 3\end{array}$ & $\begin{array}{c}B i d, B c l-2 \\
B c l-x L\end{array}$ & $\begin{array}{l}\text { Jun kinase, Fos, } \\
\text { Stat1, Nf-кb, } \\
\text { cJun, Irf-3, Irf-5 }\end{array}$ & $\begin{array}{c}\text { Rnase I, Pkr } \\
\text { 2'5' OAS }\end{array}$ & $\begin{array}{l}\text { Pea-15, } \\
\text { TGF- } \beta, \text { Ifn- } \gamma\end{array}$ \\
\hline $0.1-0.5$ & \multicolumn{7}{|c|}{ Egr-1 } \\
\hline $\begin{array}{l}\text { Three of } 6 \mathrm{n} \\
\text { RT-PCR ass } \\
\text { antiviral acti } \\
\text { The fold diff } \\
\text { lcam-1 were } \\
\text { array. Analy } \\
\text { (10- to } 100- \\
\text { tor binding }\end{array}$ & $\begin{array}{l}\text { C57BL/6 mice wer } \\
\text { vas performed to d } \\
\text { which were norma } \\
\text { ce between the me } \\
\text { asured separately } \\
\text { genes were sorted } \\
\text { "slightly increased } \\
\text { in } 5 \text {; Irf-7, interfero }\end{array}$ & $\begin{array}{l}\text { reated with } 500 \\
\text { rmine expressio } \\
\text { an to the housek } \\
\text { a were not includ in } \\
\text { cording to the ex } \\
\text { 2- to } 10 \text {-fold); "no } \\
\text { egulatory factor }\end{array}$ & $\begin{array}{l}\text { oly }(\mathrm{l}: \mathrm{C}) \text {. After } 24 \\
\text { vels of genes inv } \\
\text { ng gene } 18 \mathrm{SN} \\
\text { ive mice and the } \\
\text { on a gene array ( } \\
\text { ssion difference b } \\
\text { fected" (0.5- to } 2-\end{array}$ & $\begin{array}{l}\text { urs, livers were r } \\
\text { ed in T cell homin } \\
\text { see Methods). Th } \\
\text { edian expression } \\
\text { data are shown } \\
\text { ween naive and p } \\
\text { d); and "slightly d }\end{array}$ & $\begin{array}{l}\text { oved, and mRNA w } \\
\text { inflammation, T cell } \\
\text { ty-eight of } 48 \text { genes } \\
3 \text { poly(I:C)-treated } \\
\text { Figure } 3 A) \text {. All othe } \\
\text { y(l:C)-treated mice } \\
\text { reased" (0.1- to } 0.5\end{array}$ & $\begin{array}{l}\text { sisolated. For } \\
\text { urvival, apopto } \\
\text { howed measura } \\
\text { ice was calcula } \\
\text { genes were ana } \\
\text { id designated a } \\
\text { ld). Igfbp5, ins }\end{array}$ & $\begin{array}{l}\text { individual liver, } \\
\text { ignaling, and } \\
\text { ene expression } \\
\text { Vcam-1 and } \\
\text { in an RT-PCR } \\
\text { ghly increased" } \\
\text { ke growth fac- }\end{array}$ \\
\hline
\end{tabular}

$\mathrm{CD}^{+} \mathrm{T}$ cells (Figure 3E), suggesting binding of its ligand CXCL9 and consequent downregulation of CXCR3 (23). To analyze homing of gp33-specific cells, we transferred $10^{7}$ TCR-Tg 318 splenocytes into C57BL/ 6 recipients, followed by immunization with gp33 on days 0 and 4 together with $C \mathrm{pG}$, with or without additional poly(I:C) treatment on day 7. Again, poly(I:C) treatment induced an upregulation of $\mathrm{Cxcl} 9$ and $V \mathrm{cam}-1$, suggesting that peptide treatment had no influence on CXCL9 and VCAM-1 upregulation in the liver (Figure 3, F and G). After peptide priming, most gp33-specific cells were found to express CXCR3, which was also downregulated following poly(I:C) treatment (Figure $3 \mathrm{H}$ ). gp33-specific $\mathrm{CD}^{+} \mathrm{T}$ cells were recruited from the blood twelve hours after poly(I:C) treatment (Figure 3I) and were found within the liver in increased numbers in Alb-1 mice (Figure 3J). Together these data suggest that an innate immune activation by poly(I:C) recruits CD8 T cells into the liver, which subsequently leads to liver damage.

Role of innate signals in virus-induced hepatitis. To investigate the role of IFN- $\alpha$ in virus-induced hepatitis, we crossed the Alb-1 onto Ifnar ${ }^{/-}$mice. A total of $10^{7}$ TCR-Tg 318 splenocytes were transferred on day -1 into Alb- $1^{+}$Ifnar ${ }^{--}$mice or littermate control mice (Alb-1 $1^{+}$Ifnar ${ }^{+/-}$or Alb- $1^{+}$Ifnar ${ }^{+/+}$), followed by infection with 200 PFU LCMV on day 0 . Lack of IFN- $\alpha$ signaling resulted in an inability to clear the virus (Figure 4A), and as a consequence all mice died of generalized immune pathology by day 7 (data not shown). The transferred $\mathrm{CD}^{+} \mathrm{T}$ cells expressing type I interferon receptor could raise a functional $\mathrm{T}$ cell response (24) (Figure 4B). In littermate control mice, immunohistologically demonstrated $\mathrm{T}$ cell infiltrations in the periportal liver areas correlated with increased serum bilirubin concentrations, reflecting liver damage. Despite high viral load in the liver, LCMV-infected Alb-1+ Ifnar-- mice demonstrated a reduced infiltration of $\mathrm{T}$ cells into periportal liver areas (Figure 4A), correlating well with reduced $\mathrm{Cxcl} 9$ expression within the liver of Ifnar/- mice (Figure 4D). The lack of T cell infiltration into the periportal area of the liver also correlated with reduced serum bilirubin concentrations (Figure 4C).

We next tested whether $\mathrm{T}$ cell migration into the liver could be inhibited while preserving the beneficial antiviral function of IFN- $\alpha$.
For this purpose, we used pertussis toxin (PTX), which acts as a inhibitor of all G protein-coupled chemokine receptors (23). PTXtreated, LCMV-infected mice no longer displayed CD8 ${ }^{+} \mathrm{T}$ cell accumulation within the liver (Figure 4E), did not develop liver disease (Figure 4F), and showed limited virus antigen in the liver (Figure $4 \mathrm{E})$. To rule out the possibility that PTX treatment affected CD8 ${ }^{+}$ $\mathrm{T}$ cell priming, we investigated the ability of $\mathrm{CD} 8^{+} \mathrm{T}$ cells isolated from control or PTX-treated mice to lyse gp33-loaded target cells in vitro. PTX treatment did not alter the CTL activity of gp33-specific $\mathrm{CD}^{+} \mathrm{T}$ cells (Figure 4G). Analysis of gp33-specific $\mathrm{CD}^{+} \mathrm{T}$ cells indicated that PTX-treated Alb-1 mice contained more circulating cells expressing high levels of the IL-7 receptor $\alpha$ (IL-7R $\alpha$ ) chain (Figure 4H). Since IL-7R $\alpha$ downregulation is indicative of recent antigenic stimulation (25), these data imply that gp33-specific $\mathrm{CD}^{+} \mathrm{T}$ cells in Alb-1 mice receiving PTX did not interact with their antigen, likely resulting from the lack of migration into the liver.

\section{Discussion}

The immunoprivileged status of the liver may be built of more than one mechanism. Immune destruction of the liver requires first the priming of liver-specific T cells. These activated T cells then have to migrate into the target organ, where autoimmunity finally occurs. $\mathrm{T}$ cell priming has been proposed to be controlled by costimulatory factors, innate immune signals, and Tregs $(26,27)$. The findings presented here strongly suggested that there exists a second line of immunoprivilege in the liver. Usually, the liver does not attract liverspecific $T$ cells because chemokines are expressed at low levels in the liver. Thus, even though highly active liver-specific T cells were found at high frequencies in the blood, few migrated into the liver. Our present study further demonstrates that proinflammatory signals derived from ligation of TLR3 can lead to homing of $\mathrm{CD}^{+} \mathrm{T}$ cells to the liver with subsequent enhancement of liver disease. The mechanism by which TLR3 signaling promoted disease involved IFN- $\alpha$ - and TNF- $\alpha$-dependent upregulation of genes and their products involved in $\mathrm{T}$ cell homing and migration. This pathogenic mechanism may explain earlier observations that autoimmunity, including hepatitis, was promoted by Listeria infection (10). 
A
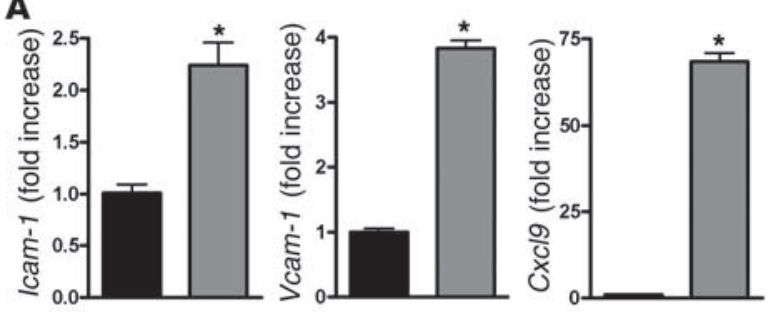

C
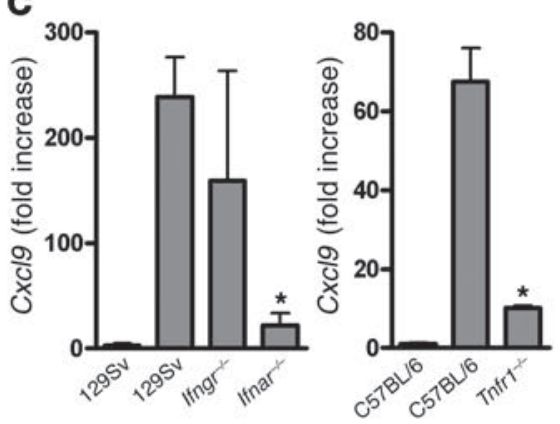

D

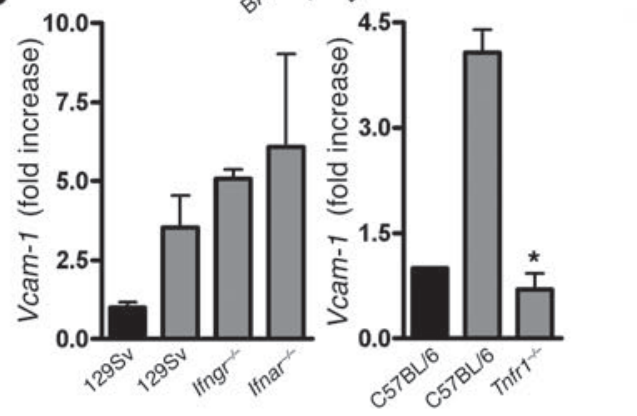

E
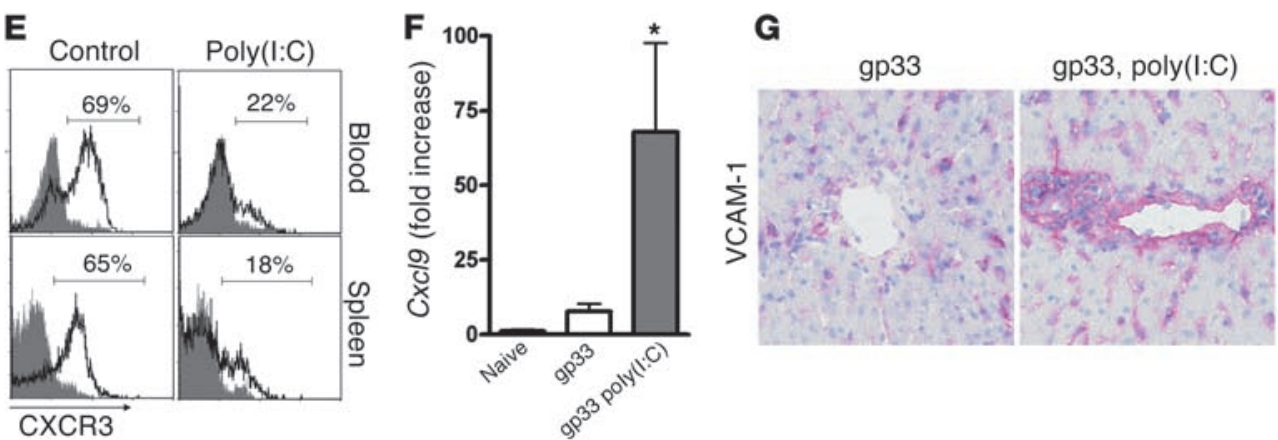

$\mathbf{H}$

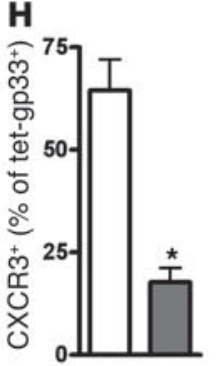

B
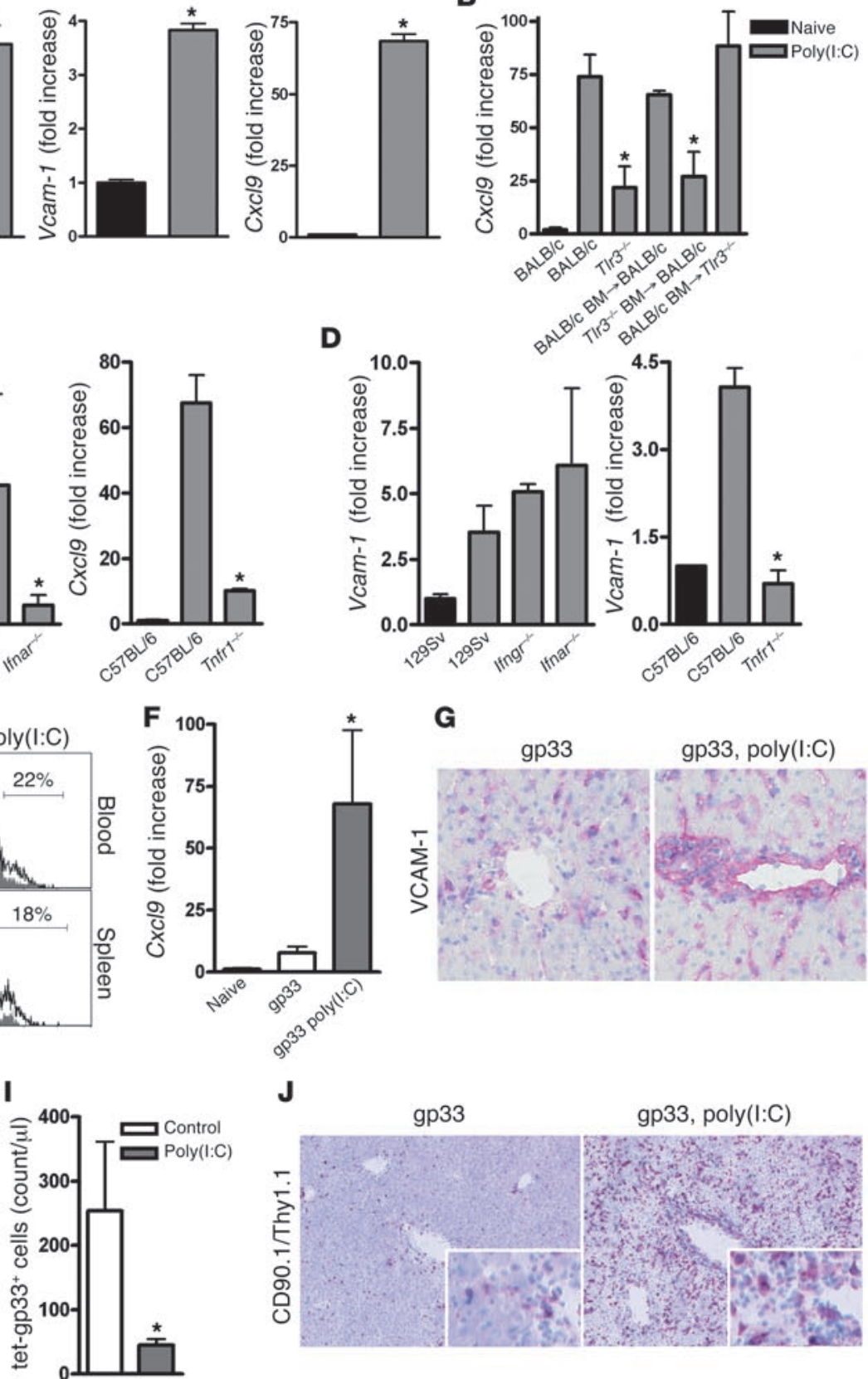

J

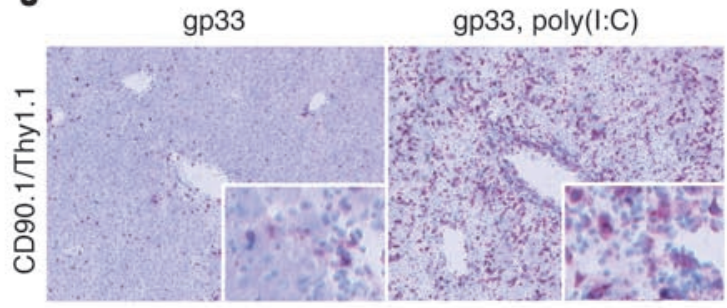

Figure 3

Activation of TLR3 and regulation of Icam-1, Vcam-1, and Cxc/9. (A-E) Mice were treated with poly(l:C). After 24 hours, RT-PCR analysis was performed in livers from C57BL/6 mice $(n=3)$ for lcam-1, Vcam-1, and Cxc/9 (A); $C x c / 9$ in livers from $B A L B / C$ and

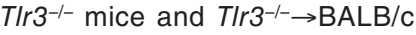
and $\mathrm{BALB} / \mathrm{C} \rightarrow$ TIr $^{-/-}$bone marrow chimeras (B); $\mathrm{Cxc} / 9$ (C) or Vcam-1 (D) in livers from Ifnar ${ }^{-1}$, Ifngr ${ }^{-1}$, and Tnfr $^{-1-}$ mice plus corresponding wild-type controls $(n=3)$. (E) Eight hours after poly(I:C) treatment, blood and spleen cells of C57BL/6 mice were analyzed for surface expression of CXCR3 by flow cytometry. Histogram plots show $\mathrm{CD}^{+}{ }^{+} \mathrm{T}$ cells gated for low CD44 expression (naive $\mathrm{CD}^{+} \mathrm{T}$ cells, gray shaded area) or high CD44 expression (memory CD8 ${ }^{+} \mathrm{T}$ cells, black line; $15 \%-20 \%$ of all CD8 ${ }^{+}$ $\mathrm{T}$ cells). (F-J) $10^{7}$ splenocytes from LCMV-gp33/H-2Db-specific TCR-Tg 318 mice were injected i.v. into $\mathrm{C} 57 \mathrm{BL} / 6$ mice $(\mathbf{F}-\mathbf{I})$ or Alb-1 mice (J) on day -1 . Recipients were then immunized with gp33 (1 mg in PBS) together with $\mathrm{CpG}$ on days 0 and 4 . One group of mice was additionally treated with poly $(I: C)$ on day 7 . On day 8 , livers were analyzed for $\mathrm{Cxc} / 9$ by RT-PCR (F) or VCAM-1 by histology (magnification, $\times 200$ ) (G). Ten hours after poly $(\mathrm{I}: \mathrm{C})$ treatment, mice were analyzed for expression of CXCR3 on tet-gp33+ ${ }^{+} D 8^{+}$ splenocytes $(\mathbf{H})$ and for absolute numbers of tet-gp33+ CD8+ T cells in the blood (I). (J) Livers of immunized Alb-1 mice were analyzed for the presence of autoreactive T cells using anti-CD90.1/Thy1.1 antibody (magnification, $\times 50$, $\times 200)$. ${ }^{*} P<0.05$.
Our findings are in accordance with previous observations indicating that expression of CXCR3 on T cells is required for the onset of autoimmune diabetes (28) and implicate TLR-induced chemokine production as a mechanism by which inflammation can be generated, resulting in exacerbated hepatitis $(29,30)$.

The most dramatic effect of TLR ligation on liver cells was the upregulation of CXCL9, which was dependent on production of type I interferon and TNF- $\alpha$. These findings suggest that treatment of chronic HCV infection with interferon (31) may enhance infiltration of $T$ cells in the liver and that although this may help to reduce viral load, it could aggravate liver destruction and cirrhosis (32) or accelerate the course of recurrent disease after transplantation.

We found that PTX-mediated inhibition of chemokine signaling provoked infiltration of only a small number of $\mathrm{T}$ cells and strongly reduced liver enzyme release and serum bilirubin concentrations. Virus was limited but still detectable. This finding correlates with a genetic polymorphism characterized by reduced chemokine signaling and chronic HCV infection in humans (33). From this data we would suggest that the inhibition of chemokine signaling in combination with interferon therapy might be a possible strategy for the treatment of chronic infection with HBV or $\mathrm{HCV}$ or disease recurrence following liver transplantation.

Tregs have been proposed to play an important role in the prevention of autoimmune disease and inflammatory processes (34). Here we have demonstrated a rise in the number of $\mathrm{CD} 25^{+} \mathrm{FoxP} 3^{+} \mathrm{CD} 4^{+}$ $\mathrm{T}$ cells after LCMV infection in Alb-1 mice (Supplemental Figure 4). Also, poly(I:C) treatment slightly increased the number of Tregs in the liver (Supplemental Figure 4). While the increase in Tregs 

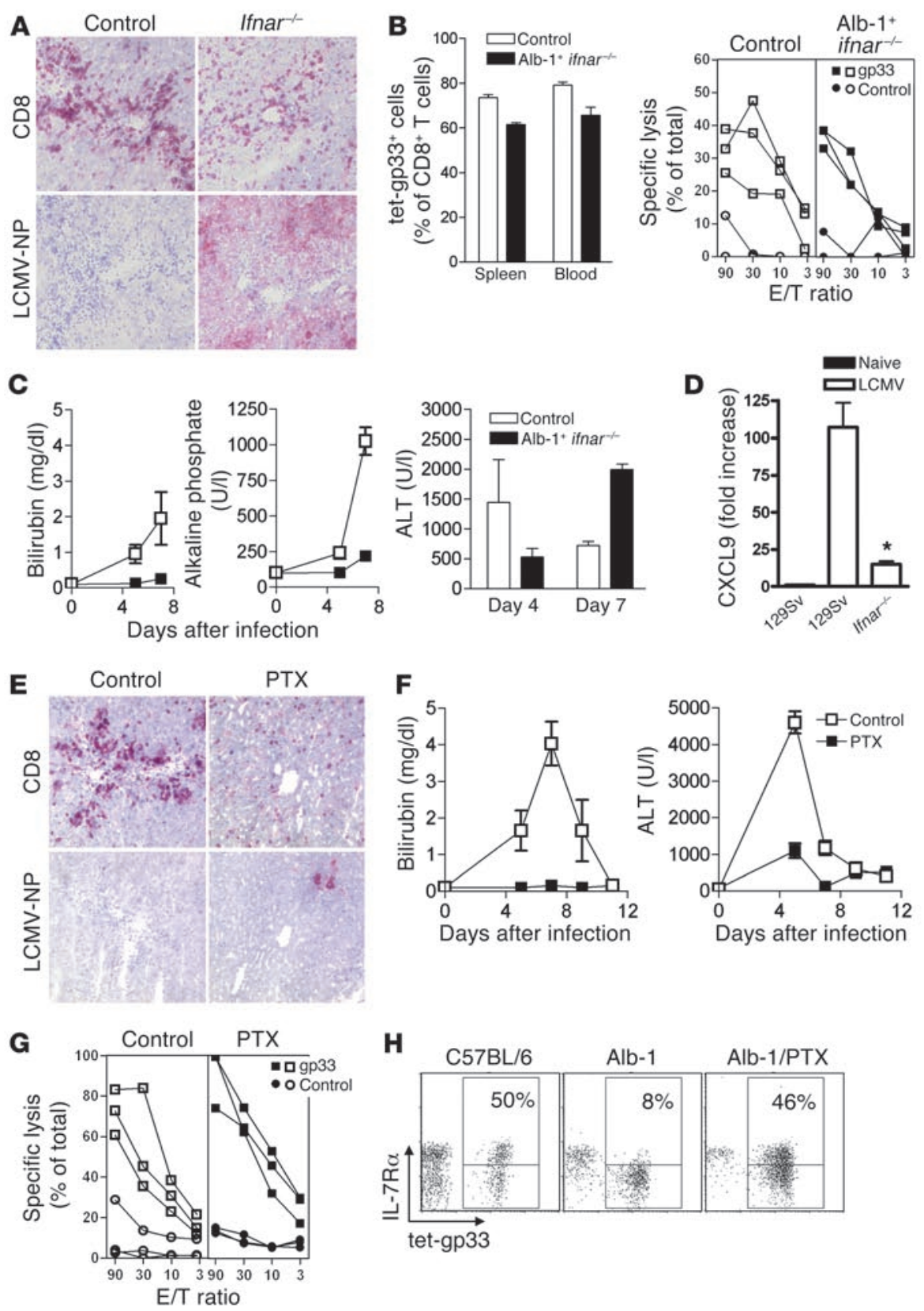

\section{Figure 4}

Homing is crucial for LCMV-induced autoimmune hepatitis. (A-C) $10^{7}$ splenocytes from

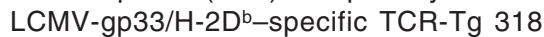
mice were transferred i.v. into Alb-1+ Ifnar ${ }^{-1}$ mice (C57BL/6 background) and littermate controls (Alb-1+ Ifnar ${ }^{+/-}$or Alb-1+ Ifnar ${ }^{+/+}$) on day -1 . Recipients were immunized with 200 PFU LCMV on day 0. (A) On day 7, Alb-1+ Ifnar ${ }^{-1-}$ mice died, and livers were analyzed for CD8 ${ }^{+} \mathrm{T}$ cell infiltration and LCMV nucleoprotein (LCMV-NP) by immunohistology (magnification, $\times 100$ ). (B) $\mathrm{CD}^{+} \mathrm{T}$ cells were analyzed by tetramer staining and in an in vitro CTL assay. (C) Serum was analyzed for bilirubin concentrations, alkaline phosphatase activity (a marker for bile cholestasis), and ALT activity ( $n=3$ per group). (D) Ifnar-1- mice (Sv129 background without transfer of $318 \mathrm{~T}$ cells) and corresponding control mice were infected with 200 PFU LCMV-WE. After 7 days, livers were analyzed for expression of CXCL9. ${ }^{\star} P>0.05$. (E-H) $10^{7}$ splenocytes from LCMV-gp33/H-2D ${ }^{\mathrm{b}}$-specific TCR-Tg 318 mice were transferred i.v. into naive Alb-1 mice or C57BL/ 6 mice on day -1 . Recipients were then infected with 200 PFU LCMV-WE on day 0 . One group was further treated with $0.5 \mu \mathrm{g}$ of PTX on days 0 and 3. (E) On day 6 , liver sections were analyzed for CD8 ${ }^{+} T$ cell infiltration or for viral antigen (LCMV$\mathrm{NP}$ ) by histology (magnification, $\times 100 ; n=2$ per group). Serum was analyzed for bilirubin concentration and ALT activity ( $n=4$ per group) (F), and CD8 ${ }^{+} \mathrm{T}$ cell effector function was assessed by an in vitro CTL assay (G). (H) Autoreactive CD8 ${ }^{+}$T cells and their IL-7R $\alpha$ expression were analyzed in the blood ( $n=4$ per group). cannot explain the induction of hepatitis, it might be required for the observed downregulation of disease after day 7 .

TNF- $\alpha$-induced CXCL9 may play a role in human hepatitis, as there is a direct link between a mutation in the TNF- $\alpha$ locus and autoimmune hepatitis (35). These clinical observations together with our data suggest that inhibition of chemokines may prove an interesting therapeutic approach against immune hepatitis in humans.

In conclusion, the presented experiments revealed that highly activated $\mathrm{CD}^{+} \mathrm{T}$ cells can "peacefully" coexist with hepatocytes expressing the relevant autoantigen without causing overt disease. Engagement of TLR3 was required to break this immunoprivileged state of the liver and converted autoreactivity into overt autoimmune disease and organ destruction. Taken together, our results have identified TLR 3 as one important mechanism of solid organ immune privilege.

\section{Methods}

Mice and viruses. LCMV strain WE was originally obtained from F. Lehmann-Grube (Heinrich Pette Institute, Hamburg, Germany) and was propagated in L929 cells. Virus titers were measured using a plaque-forming assay as described previously (36). Mice were infected with $200 \mathrm{PFU}$ LCMV-WE. Mice transgenic for a TCR recognizing LCMV-glycoprotein $33-41$

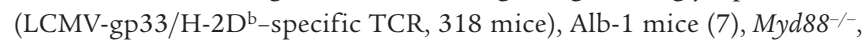
and $\mathrm{Tnfr}^{-/-}$mice were maintained on the $\mathrm{C} 57 \mathrm{BL} / 6$ genetic background, while Ifngr ${ }^{-1-}$ mice were of the $129 \mathrm{~Sv}$ background. Ifnar ${ }^{-1}$ mice were of the $129 \mathrm{~Sv}$ or of the C57BL/6 background (for crossings to Alb-1). Tlr $3^{-/-}$mice were on the $\mathrm{BALB} / \mathrm{c}$ background. For generation of bone marrow chimeras, recipient mice were irradiated with 9.50 Gy on day -1 . On day $0,10^{7}$ bone marrow cells were transferred i.v., and mice were used for experiments 7 weeks later. All experiments were performed with animals housed in ventilated cages. Animal experiments were approved and authorized by 
the cantonal Veterinär office (Zurich, Switzerland) and in accordance with Swiss animal protection law.

PTX treatment. For inhibition of $\mathrm{G}$ protein-coupled chemokine signaling, $0.5 \mu \mathrm{g}$ PTX (Sigma-Aldrich) was administered i.v. on days 0 and 3.

Immunization with peptides or TLR ligands and adoptive transfer of CD8 $8^{+} T$ cells. gp33 peptide (KAVYNFATM) was synthesized by Neosystems. For priming of LCMV-gp33/H-2D $\mathrm{D}^{\mathrm{b}}$-specific TCR-Tg CD8 ${ }^{+} \mathrm{T}$ cells, $10^{7}$ splenocytes were adoptively transferred to Alb- 1 mice or C57BL/ 6 mice on day -1 , and mice were immunized with $1 \mathrm{mg}$ gp33 together with CpG (ODN-1826) (50 $\mu \mathrm{g}$; Coley) on days 0 and 4 . To analyze the influence of TLRs, we treated mice with the TLR3 ligand poly(I:C) (500 $\mu$; Sigma-Aldrich) or the TLR9 ligand CpG (ODN-2216) (100 $\mu \mathrm{g}$; TIB MOLBIOL).

Cytotoxicity assay. ${ }^{51} \mathrm{Cr}$ release assays were performed as previously described (36) and supernatants analyzed after 8 hours. Assays for in vivo cytotoxicity were performed using C57BL/6 splenocytes incubated for 1 hour with or without gp33 and labeled for 10 minutes with $5 \mu \mathrm{g} / \mathrm{ml}$ (peptide-labeled splenocytes) or $0.5 \mu \mathrm{g} / \mathrm{ml}$ (unlabeled splenocytes) CFSE (Invitrogen), respectively. A total of $10^{7}$ cells of each fraction was injected intravenously, and the number of CFSE-positive cells remaining in the spleen after 2 hours was determined by FACS analysis.

Histology. Histological analyses were performed on snap-frozen tissue. Sections were stained with rat $\mathrm{mAbs}$ against murine MHC class I(M1/42), ICAM-1 (CD54, KAT-1; AbD Serotec), VCAM-1 (CD106, M/K-2; AbD Serotec), CD8 (53-6.7; BD Biosciences), or VL4, an mAb against the LCMV nucleoprotein. Sections were further stained with goat anti-CXCL9 (MIG; R\&D Systems) and anti-Thy1.1 antibody (CD90.1, HIS51; BD Biosciences). Staining was developed using a goat anti-rat antibody (R40000; CALTAG Laboratories) and an alkaline phosphatase-coupled donkey anti-goat antibody (705055-147; Jackson ImmunoResearch Laboratories Inc.) with naphthol AS-BI (6-bromo-2-hydroxy-3-naphtholic acid 2-methoxy anilide) phosphate and new fuchsin as a substrate. The sections were counterstained with hemalum.

Bilirubin, alkaline phosphatase, and ALT. Concentrations were measured using a serum multiple biochemical analyzer (Ektachem DTSCII; Johnson \& Johnson).

IFN- $\alpha$ ELISA. IFN- $\alpha$ ELISA was performed according to the manufacturer's protocol (Research Diagnostics Inc.).

FACS analysis. Tetramers were produced and FACS analysis were performed as described previously (36). Briefly, splenocytes or peripheral blood lymphocytes were stained using PE-labeled gp33 MHC class I tetramers $\left(\mathrm{gp} 33 / \mathrm{H}-2 \mathrm{D}^{\mathrm{b}}\right)$ for 15 minutes at $37^{\circ} \mathrm{C}$, followed by staining with anti-CD8-PerCP (BD Biosciences) for 30 minutes at $4^{\circ} \mathrm{C}$. For determination of their activation status, lymphocytes were stained with anti-CD25, anti-CD69, anti-CD62L, anti-CD44, anti-VLA-4, and anti-IL-7R $\alpha$ (BD Biosciences - Pharmingen) or anti-CXCR3 (R\&D Systems) for 30 minutes at $4^{\circ} \mathrm{C}$. For staining of Tregs, anti-FoxP3 antibody was used (eBioscience).

$m R N A$ gene profiling by quantitative RT-PCR. Total RNA was extracted from liver tissue using TRIzoL reagent (Invitrogen) following the manufacturer's instructions. RNA was transcribed using ThermoScript RT-PCR System kit (Invitrogen) or a 1-step RT-PCR kit (Applied Biosystems). Predesigned TaqMan low-density arrays (format 48x8 [part no. 4342253], micro fluidic cards; Applied Biosystems) were used in a 2-step RT-PCR process using the ABI Prism 7900HT Sequence Detection System (Applied Biosystems) with a TaqMan Low Density Array Upgrade (Applied Biosystems). Loading of the low-density arrays, standard thermal cycling conditions, and data acquisition were done according to the manufacturer's suggestions. Quantitative real-time PCR amplification of single genes was performed using an ABI Prism 7000 Sequence Detector System (Applied Biosystems). TaqMan gene expression assays (Applied Biosystems) for TNF- $\alpha$ (assay ID Mm 00443258_m), VCAM-1 (assay ID Mm 00449197_m1), ICAM-1 (assay ID Mm00516023_m1), and Cxcl9 (assay ID Mm00434946_m1) were used for quantification of mRNA expression of the respective genes. For analysis, expression levels of target genes were normalized to the housekeeping gene $18 \mathrm{~S}$ rRNA $(\Delta \mathrm{Ct})$ or UBE2D2. Gene expression values were then calculated based on the $\Delta \Delta C$ t method, with data for all samples analyzed against the mean value for 3 untreated mice. Relative quantities (RQ) were determined using the equation: $R Q=2^{-\Delta \Delta \mathrm{Ct}}$.

Statistics. Data are expressed as mean \pm SEM. For statistical analysis, 1 -tailed Student's $t$ test was used, and $P$ values $<0.05$ were considered statistically significant.

\section{Acknowledgments}

We would like to thank Adriano Aguzzi for suggestions, discussions, and critical comments. We are also grateful to Kathrin Tschannen for technical support; Antje Nowotny, Silvia Behnke, and André Fitsche for excellent histological analysis; and Michele Bernasconi and the Department of Oncology, University Children's Hospital in Zurich, for providing access to an ABI PRISM $7900 H T$ Sequence Detection System. This study was supported by the Swiss National Science Foundation and Deutsche Forschungsgemeinschaft (DFG) LA1419/1-1.

Received for publication February 27, 2006, and accepted in revised form June 27, 2006.

Address correspondence to: Karl Lang, Institute of Experimental Immunology, University Hospital, Schmelzbergstrasse 12, CH8091 Zurich, Switzerland. Phone: 41-1-255-2734; Fax: 41-1-2554420; E-mail: karl.lang@usz.ch.

Tobias Junt's present address is: CBR Institute for Biomedical Research, Harvard Medical School, Boston, Massachusetts, USA.

Karl S. Lang, Panco Georgiev, and Mike Recher contributed equally to this work.
1. Kamada, N., Davies, H.S., and Roser, B. 1981. Reversal of transplantation immunity by liver grafting. Nature. 292:840-842.

2. Bumgardner, G.L., and Orosz, C.G. 2000. Unusual patterns of alloimmunity evoked by allogeneic liver parenchymal cells. Immunol. Rev. 174:260-279.

3. Diamantis, I., and Boumpas, D.T. 2004. Autoimmune hepatitis: evolving concepts. Autoimmun. Rev. 3:207-214.

4. Okano, N., et al. 2003. Clinicopathological features of acute-onset autoimmune hepatitis. Hepatol. Res. 25:263-270.

5. Luxon, B.A. 2003. Autoimmune hepatitis. Making sense of all those antibodies. Postgrad. Med. 114:79-82, 85-88.

6. Horwitz, M.S., et al. 1998. Diabetes induced by
Coxsackie virus: initiation by bystander damage and not molecular mimicry. Nat. Med. 4:781-785.

7. Voehringer, D., et al. 2000. Break of T cell ignorance to a viral antigen in the liver induces hepatitis. J. Immunol. 165:2415-2422.

8. Ferber, I., et al. 1994. Levels of peripheral T cell tolerance induced by different doses of tolerogen. Science. 263:674-676.

9. Hammerling, G.J., Schonrich, G., Ferber, I., and Arnold, B. 1993. Peripheral tolerance as a multistep mechanism. Immunol. Rev. 133:93-104.

10. Limmer, A., et al. 1998. Failure to induce organspecific autoimmunity by breaking of tolerance: importance of the microenvironment. Eur. J. Immunol. 28:2395-2406.

11. Sacher, T., et al. 2002. CpG-ODN-induced inflam- mation is sufficient to cause T-cell-mediated autoaggression against hepatocytes. Eur. J. Immunol. 32:3628-3637.

12. Akira, S., and Takeda, K. 2004. Toll-like receptor signalling. Nat. Rev. Immunol. 4:499-511.

13. Devendra, D., et al. 2005. Interferon-alpha as a mediator of polyinosinic:polycytidylic acid-induced type 1 diabetes. Diabetes. 54:2549-2556.

14. Lang, K.S., et al. 2005. Toll-like receptor engagement converts T-cell autoreactivity into overt autoimmune disease. Nat. Med. 11:138-145.

15. Bauer, S., et al. 2001. Human TLR9 confers responsiveness to bacterial DNA via species-specific CpG motif recognition. Proc. Natl. Acad. Sci. U. S. A. 98:9237-9242.

16. Alexopoulou, L., Holt, A.C., Medzhitov, R., and 
Flavell, R.A. 2001. Recognition of double-stranded RNA and activation of NF-kappaB by Toll-like receptor 3. Nature. 413:732-738.

17. Zhou, S., et al. 2005. MyD88 is critical for the development of innate and adaptive immunity during acute lymphocytic choriomeningitis virus infection. Eur. I. Immunol. 35:822-830.

18. Von Herrath, M.G., and Oldstone, M.B. 1997. Interferon-gamma is essential for destruction of beta cells and development of insulin-dependent diabetes mellitus. J. Exp. Med. 185:531-539.

19. Chen, M., Tabaczewski, P., Truscott, S.M., Van Kaer, L., and Stroynowski, I. 2005. Hepatocytes express abundant surface class I MHC and efficiently use transporter associated with antigen processing, tapasin, and low molecular weight polypeptide proteasome subunit components of antigen processing and presentation pathway. J. Immunol. 175:1047-1055.

20. Shiow, L.R., et al. 2006. CD69 acts downstream of interferon-alpha/beta to inhibit S1P1 and lymphocyte egress from lymphoid organs. Nature. 440:540-544.

21. Bisikirska, B., Colgan, J., Luban, J., Bluestone, J.A., and Herold, K.C. 2005. TCR stimulation with modified anti-CD $3 \mathrm{mAb}$ expands $\mathrm{CD}^{+} \mathrm{T}$ cell popula- tion and induces $\mathrm{CD}^{+} \mathrm{CD} 25^{+}$Tregs. J. Clin. Invest. 115:2904-2913. doi:10.1172/JCI23961.

22. Yoneyama, M., et al. 2004. The RNA helicase RIG-I has an essential function in double-stranded RNAinduced innate antiviral responses. Nat. Immunol. 5:730-737.

23. Thelen, M. 2001. Dancing to the tune of chemokines. Nat. Immunol. 2:129-134.

24. Kolumam, G.A., Thomas, S., Thompson, L.J. Sprent, J., and Murali-Krishna, K. 2005. Type I interferons act directly on CD8 T cells to allow clonal expansion and memory formation in response to viral infection. J. Exp. Med. 202:637-650.

25. Lang, K.S., et al. 2005. Inverse correlation between IL-7 receptor expression and CD8 T cell exhaustion during persistent antigen stimulation. Eur. J. Immunol. 35:738-745.

26. Stager, S., and Kaye, P.M. 2004. CD8+ T-cell priming regulated by cytokines of the innate immune system. Trends Mol. Med. 10:366-371.

27. Bharat, A., Fields, R.C., and Mohanakumar, T. 2005. Regulatory T cell-mediated transplantation tolerance. Immunol. Res. 33:195-212.

28. Frigerio, S., et al. 2002. Beta cells are responsible for CXCR3-mediated T-cell infiltration in insulitis. Nat. Med. 8:1414-1420.
29. Aceti, A., Mura, M.S., Babudieri, S., and Bacciu, S.A. 1995. A young woman with hepatitis after a sore throat. Lancet. 346:1603.

30. Mieli-Vergani, G., Sutherland, S., and Mowat, A.P. 1989. Measles and autoimmune chronic active hepatitis [letter]. Lancet. 2:688.

31. Kim, A.I., and Saab, S. 2005. Treatment of hepatitis C. Am. J. Med. 118:808-815.

32. Garcia-Buey, L., et al. 1995. Latent autoimmune hepatitis triggered during interferon therapy in patients with chronic hepatitis C. Gastroenterology. 108:1770-1777.

33. Promrat, K., et al. 2003. Associations of chemokine system polymorphisms with clinical outcomes and treatment responses of chronic hepatitis C. Gastroenterology. 124:352-360.

34. Maloy, K.J., et al. 2003. CD4+CD25+ T(R) cells suppress innate immune pathology through cytokine-dependent mechanisms. J. Exp. Med. 197:111-119.

35. Cookson, S., et al. 1999. Frequency and nature of cytokine gene polymorphisms in type 1 autoimmune hepatitis. Hepatology. 30:851-856.

36. Junt, T., et al. 2002. Antiviral immune responses in the absence of organized lymphoid $\mathrm{T}$ cell zones in plt/plt mice. J. Immunol. 168:6032-6040. 